\title{
Kindgerechte Therapie bei pulmonaler arterieller Hypertonie
}

_ Die pulmonale arterielle Hypertonie $(\mathrm{PAH})$ ist definiert als Anstieg des mittleren Pulmonalarteriendrucks in Ruhe auf mehr als $25 \mathrm{~mm} \mathrm{Hg}$. Sie liegt bei Kindern und Jugendlichen meist aufgrund eines sekundären Herzfehlers als sekundäre PAH vor Die Prognose ist in dieser Altersgruppe besonders schlecht: Bleibt die Erkrankung nach der Diagnosestellung unbehandelt, liegt die geschätzte mittlere Überlebensdauer bei nur zehn Monaten.

2009 wurde europaweit eine speziell für die Behandlung pädiatrischer PAH-Formen entwickelte teil- und dispergierbare Tablette mit dem Wirkstoff Bosentan zugelassen. Das Präparat (Tracleer ${ }^{\circledR}$ ) ist damit das erste und bislang einzige PAH-Therapeutikum mit geeigneter Formulierung für Kinder $a b$ zwei Jahren.

Die Zulassung beruht auf den Daten der offenen FUTURE-1-Studie. In diese multizentrische Phase-III-Untersuchung wur- den 36 Kinder im Alter zwischen zwei und zwölf Jahren eingeschlossen, die an idiopathischer oder erblicher PAH erkrankt waren. Vier Wochen lang erhielten die Patienten zunächst zweimal täglich $2 \mathrm{mg} / \mathrm{kg}$ KG Bosentan in der neuen Darreichungsform. Die Dosis wurde dann auf zweimal täglich $4 \mathrm{mg} / \mathrm{kg}$ in den darauf folgenden Wochen erhöht. Die pharmakokinetischen Daten zeigten unter beiden Dosierungen vergleichbare Plasmakonzentrationen. Die empfohlene Tagesdosis beträgt daher laut Fachinformation zweimal $2 \mathrm{mg} / \mathrm{kg}$. Bei $90 \%$ der Kinder bewerteten Ärzte und Eltern den klinischen Gesamteindruck unter der Therapie als stabil oder verbessert.

kr

Symposium „PAH bei Kindern und Jugendlichen - Therapie von Anfang an“ im Rahmen der 41. Jahrestagung der DGPK. Weimar, 4. Oktober 2009. Veranstalter: Actelion, Freiburg

\section{Inhalationsanalgesie für kurze Eingriffe}

\begin{abstract}
— Schmerzhafte Prozeduren wie Wundversorgung, Entfernung von Fremdmaterialien oder Repositionen sind bei Kindern häufig problematisch. Angst und Schmerzen sowie Abwehrverhalten müssen zunächst vermindert werden, um die Eingriffe sicher durchführen zu können. Distickstoffmonoxid (Lachgas) ist ein seit rund 160 Jahren bewährter Wirkstoff, der kurzfristig analgetisch und beruhigend wirkt und sich daher bei kurzen schmerzhaften Eingriffen gut eignet. Die Gabe von Lachgas und reinem Sauerstoff im Verhältnis von 50:50 ist bei sachgerechter Anwendung sicher, betonte Dr. Georg Staubli aus Zürich. Seit 2008 ist diese Fertigmischung in Deutschland unter dem Namen LIVOPAN ${ }^{\circledR}$ für die Behandlung von kurzzeitigen Schmerzzuständen leichter bis mittlerer Intensität zugelassen.

Die Patienten halten sich die Inhalationsmaske meist selbst vor Mund und Nase, bei kleineren Kindern kann dies auch die Begleitperson übernehmen. Durch die Selbstapplikation des Analgetikums erhielten
\end{abstract}

die Patienten das Gefühl der Kontrolle und seien vom eigentlichen Eingriff abgelenkt, so Staubli, der seit Jahren das Lachgas-Sauerstoff-Gemisch routinemäßig einsetzt. Die mittelstarke analgetische und sedierende Wirkung ist bereits nach zwei bis drei Minuten spürbar und bleibt erhalten, solange das Gas eingeatmet wird. Häufigste Nebenwirkungen sind Schwindel und leichte Übelkeit, die nach Ende der Inhalation rasch wieder abklingen. Da Lachgas unverändert ausgeatmet wird, sollte die Atemluft des Patienten über einen Schlauch z. B. durch ein geöffnetes Fenster abgeführt werden. Sind auch nach dem Eingriff noch Schmerzen zu erwarten, sollte dem Patienten bereits 30 bis 60 Minuten vor dem Eingriff ein orales Analgetikum wie Ibuprofen oder Paracetamol gegeben werden.

bk

Satellitensymposium „LIVOPAN ${ }^{\circledR}$ bei kurzen schmerzhaften Eingriffen" im Rahmen der 22. Norddeutschen Anästhesie-Tage.

Hamburg, 11. Dezember 2009. Veranstalter: Linde Gas Therapeutics, Unterschleißheim
Aknetherapie ohne Antibiotika

Bei der Behandlung der Akne werden häufig Antibiotika eingesetzt. Allerdings ist dabei die Entwicklung multiresistenter bakterieller Erreger alarmierend. Nicht nur die nachlassende Sensibilität von Propionibakterien ist bei einer antibiotikahaltigen Aknetherapie die Folge, da Antibiotika niemals spefizisch gegen nur eine bakterielle Spezies wirken, können sie auch das therapeutische Armentarium bei schwerwiegenden systemischen Infektionen einschränken. Experten mahnen daher zur Zurückhaltung bei der Verordnung von Antibiotika.

Vor diesem Hintergrund gewinnen antibiotikafreie Therapieansätze in der Aknetherapie immer mehr an Bedeutung. Die Kombination aus dem Retinoid Adapalen und Benzoylperoxid (Epiduo ${ }^{\circledR}$ ) ist eine wirkungsvolle Behandlungsalternative. Benzoylperoxid ist bakterizid, während Adapalen eine antiinflammatorische, antikomedogene sowie komedolytische Wirkung zeigt. Das Präparat eignet sich zur Akut- wie auch zur Erhaltungstherapie.

Nach Informationen von Galderma, Düsseldorf

\section{Diabetes im Blickpunkt}

Vom Typ-1-Diabetes ist in Deutschland bereits eines von 600 Kindern betroffen, die Neuerkrankungsrate steigt jährlich um $3-4 \%$. Noch dramatischer ist der Anstieg beim Typ-2-Diabetes: Jährlich wird er bereits bei ca. 200 Jugendlichen diagnostiziert und bei vielen ein gestörter Kohlenhydratstoffwechsel. Im Rahmen seines Konzeptes „Changing Diabetes Diabetes verändern“ unterstützte der Diabetesspezialist Novo Nordisk im Jahr 2009 Aktionen, bei denen Kinder altersgerecht über Nahrungsmittel aufgeklärt und betroffene Kinder im Umgang mit ihrer Krankheit unterstützt wurden. Dies galt etwa für die Aufklärung von über 500 Schulklassen und Kindergartengruppen über Zucker in Lebensmitteln und gesunde Ernährung. Eine andere Aktion war der Malwettbewerb „Bilder erzählen meine Krankheit“, bei dem Kinder sich bildhaft mit dem Diabetes auseinandersetzen und ihre Gefühle und Ansichten ausdrücken konnten.

Nach Informationen von Novo Nordisk, Mainz 